American Journal of Animal and Veterinary Sciences 5 (1): 45-51, 2010

ISSN 1557-4555

(C) 2010 Science Publications

\title{
Efficacy and Characteristics of different Methods of Coccidiosis Infection in Broiler Chickens
}

\author{
${ }^{1}$ M.A. Elmusharaf, ${ }^{2}$ H.E. Mohamed, ${ }^{2}$ A. Alhaidary and ${ }^{1,2}$ A.C. Beynen \\ ${ }^{1}$ Department of Nutrition, Faculty of Veterinary Medicine, Utrecht University, The Netherlands \\ ${ }^{2}$ Department of Animal Production, College of Food and Agricultural Sciences, \\ King Saud University, Riyadh, Kingdom of Saudi Arabia
}

\begin{abstract}
Problem statement: Different methods of experimental infection of broiler chickens with Eimeria species have been described in the literature. These methods had not been compared and contrasted so as to contribute to the selection of the most appropriate model of coccidiosis in broiler chickens. Identifying such a model was important to speed up the screening of potential coccidiostatics. Approach: In five different experiments with broiler chickens, we used different methods of infection with Eimeria species. In this paper the different methods and the results have been evaluated. Results: Administration through gavage into the crop of relatively low doses of either Eimeria tenella alone, or in combination with Eimeria acervulina and Eimeria maxima, did not influence body-weight gain and feed intake, but did induce intestinal lesions and faecal shedding of oocysts. The administration of an identically high number of sporulated oocysts in the form of a mixture of the three Eimeria species, either through a single dose by gavage or through the litter, produced similar lowering effects on body-weight gain or feed intake, similar degrees of severity of intestinal lesions and similar rates of faecal oocyst shedding. Conclusion: Depending on the variables considered of interest, the present data may indicate the most appropriate model. The model using infection with oocysts through the litter may optimally mimic the field situation in combination with controlled conditions and allowing experimental flexibility and a high number of experimental units within the research facility.
\end{abstract}

Key words: Infection models, coccidia, broilers

\section{INTRODUCTION}

Coccidiosis is an infectious disease caused by the protozoan parasite of the genus Eimeria (Tyzzer et al., 1932). The disease is almost universal in poultry production and E. acervulina, E. brunetti, E. maxima, E. mitis, E. necatrix, E. praecox and E. tenella are considered as the pathogenic species (Dalloul and Lillehoj, 2006). Infection with coccidiosis follows the ingestion of viable oocysts, which are contaminants of food, dust and water. After the oocysts are swallowed, the sporozoites are liberated and a life cycle in the host tissues begins, leading to the faecal excretion of newly formed oocysts. Coccidiosis remains one of the most expensive and common diseases in poultry production (Dalloul and Lillehoj, 2006).

Given the importance of coccidiosis in poultry production, consumer concern and the expected ban on the use of coccidiostatics, there is great interest in the development of alternatives in the prevention and treatment of the disease. In order to study the efficacy of alternatives, a poultry model of coccidiosis is needed. In the course of our studies on alternative approaches to counteract coccidiosis in broiler chickens, we have used different models of infection in four different experiments. In this study, we compare and contrast the various methods that we have used (Elmusharaf et al., 2006, 2007a; 2007b). It is anticipated that the data presented here may contribute to the selection of the most appropriate infection model of coccidiosis in broiler chickens.

\section{MATERIALS AND METHODS}

Animals, diet and experimental designs: One-day-old broiler chicks (Ross 308) were purchased from a local hatchery. On arrival (day 1) they were wing-banded, weighed and randomly allocated to the treatments. The birds were housed in wire-suspended cages equipped with plastic sides. The cages had either their wire floor

Corresponding Author: M.A. Elmusharaf, Department of Nutrition, Faculty of Veterinary Medicine, Utrecht University, The Netherlands 
as such (Experiment 1 and 2) or covered with plastic and clean wood shavings as bedding (Experiment 3-5). Ambient temperature was gradually decreased from $32^{\circ} \mathrm{C}$ on day $1-20^{\circ} \mathrm{C}$ at the end of the experiments. During the entire experimental periods there was continuous lighting. The basal diet used did not contain growth promoters or coccidiostatics. The composition was as follows ( $\mathrm{g} \mathrm{kg}^{-1}$ diet): wheat (+ xylanase), 250; corn, 321; soyabean meal (46.7\% crude protein), 225; peas, 50; sunflower meal (32\% crude protein), 40; potato protein, 15 ; fish meal ( $72 \%$ crude protein), 25 ; soyabean oil, 40; premix (Research Diet Services, Wijk bij Duurstede, The Netherlands), 5; limestone, 16; monocalcium phosphate, 7; phytase (Natuphos 5000G), 0.1 ; salt, 1.7; sodium bicarbonate, 1.7; L-lysine $\mathrm{HCl}$, 0.8; D,L-methionine, 1.7. The diets were fed ad libitum as from arrival of the birds until the end of the experiments. Tap water was freely available.

Table 1 gives a brief overview of the five experiments that were carried out. Between the experiments, there are differences in the coccidiosis infection as to time point, Eimeria species and dose and route of oocyst administration. Below the experimental designs are described in more detail.

Experiment 1: The control and infected group consisted of 5 replicates of 5 birds each. Each replicate was housed in a separate cage and all birds were fed the basal diet. On day 12, one group was inoculated with 3500 sporulated oocysts of the E. tenella (Houghton strain) laboratory strain. The oocysts were obtained from the Animal Health Service Ltd., Poultry Health Centre (Deventer, The Netherlands). The oocysts were administered with $1 \mathrm{~mL}$ of tap water via an oral gavage directly into the crop. The control group was given water only through gavage. Care was taken to prevent cross contamination throughout the experiment. At the end of the experiment (day 21), the birds were euthanized by cervical dislocation.

Experiment 2: The control and infected group consisted of 5 replicates of 3 birds each. Each replicate was housed in a separate cage and all birds were fed the basal diet. On day 8, one group of birds was inoculated with 5000 sporulated oocysts of E. tenella. The oocysts were administered as described for Experiment 1. The control group was given water through gavage. On day 14 , all birds were killed by cervical dislocation.

Experiment 3: The control and infected group consisted of 8 replicates of 8 birds each. Each replicate was housed in a separate cage and all birds were fed the basal diet. On day 1, one group was challenged with a mixture of Eimeria containing 900 sporulated oocysts of $E$. acervulina (Weybridge strain), 570 sporulated oocysts of E. maxima (Weybridge strain) and 170 sporulated oocysts of E. tenella (Houghton strain). The oocysts were laboratory strains and the dose and strains simulated commercially available live vaccines (Williams, 2002). The sporulated oocysts were administered with $1 \mathrm{~mL}$ of tap water directly into the crop via a scaled $1 \mathrm{~mL}$ syringe. The non-infected birds were given $1 \mathrm{~mL}$ of oocyst-free water into the crop. On day 14, one bird per cage per treatment was killed by cervical dislocation and used for lesion scoring. On day 19, all remaining birds were killed by cervical dislocation.

Experiment 4: The control and the infected group consisted of 10 replicates, each replicate comprising 8 birds. Each replicate was housed in a separate cage and all birds were given the basal diet. On day 15, the chickens of one group were individually challenged with a mixture of Eimeria containing $1.76 \times 10^{4}$ sporulated oocysts of E. acervulina (Weybridge strain), $1.25 \times 10^{4}$ sporulated oocysts of E. maxima (Weybridge strain) and $7.5 \times 10^{3}$ sporulated oocysts of E. tenella (Houghton strain). The sporulated oocysts were laboratory strains and administered with $1 \mathrm{~mL}$ of tap water via a scaled $1 \mathrm{~mL}$ syringe directly into the crop. The negative groups were also given $1 \mathrm{~mL}$ water via a scaled $1 \mathrm{~mL}$ syringe directly into the crop, but without oocysts. On days 21 and 28, two birds per treatment were killed by cervical dislocation and on day 35 the remaining birds were killed.

Table 1: Overview of the five experiments

\begin{tabular}{|c|c|c|c|c|}
\hline Experiment & Day of infection & Route of infection & $\begin{array}{l}\text { Eimeria species (dose of } \\
\text { sporulated oocysts/bird) }\end{array}$ & Measurements \\
\hline 1 & 12 & Gavage into crop & E. tenella $(3500)$ & Growth performance lesion scores \\
\hline 2 & 8 & Gavage into crop & E. tenella $(5000)$ & Growth performance, lesion scores, $\mathrm{OPG}^{1}$ \\
\hline 3 & 1 & Gavage into crop & $\begin{array}{l}\text { E. acervulina }(900) \\
\text { E. maxima }(570)\end{array}$ & Growth performance, lesion scores, OPG \\
\hline & & & E. tenella $(170)$ & \\
\hline 4 & 15 & Gavage into crop & $\begin{array}{l}\text { E. acervulina }(17600) \\
\text { E. maxima }(12500) \\
\text { E. tenella }(7500)\end{array}$ & Growth performance, lesion scores, OPG \\
\hline 5 & 15 & Through litter & $\begin{array}{l}\text { E. acervulina }(17600) \\
\text { E. maxima }(12500) \\
\text { E. tenella }(7500)\end{array}$ & Growth performance, lesion scores, OPG \\
\hline
\end{tabular}


Experiment 5: The control and infected group consisted of 8 replicates of 8 birds each. Each replicate was housed in a separate cage and all birds were given the basal diet. On day 15, a simulation of a natural coccidial infection within the broilers' test facility was created. The infected replicates were placed in new litter seeded with sporulated oocysts. Individual birds would pick up the oocysts from the litter in different amounts and at different times, thus mimicking a natural, progressive infection. The uninfected replicates were placed in clean litter. Sporulated oocysts of E. acervulina (Weybridge strain), E. maxima (Weybridge strain) and E. tenella (Houghton strain) laboratory strains were used. A fixed amount of each species in a mixture $\left(1.76 \times 10^{4}\right.$ E. acervulina, $1.25 \times 10^{4}$ E. maxima and $7.5 \times 10^{3}$ E. tenella per bird) were mixed into wood shavings and spread evenly across the back half of the cage. On days 21 and 28, two birds per treatment were killed by cervical dislocation and on day 35 the remaining birds were killed.

\section{Sampling and measurements:}

Technical performance: In Experiment 1 and 2, body weights were recorded on the day of infection and at 6 days Post Infection (PI). In Experiment 3-5, body weights were measured on the day of the infection, 6 days PI and then weekly until the end of each experiment. For the various time intervals, feed intakes were determined and expressed as g bird ${ }^{-1}$ day $^{-1}$. Feed Conversion Ratios (FCR) were calculated as feed intake per cage divided by weight gain of birds in the cage.

Oocyst Counting: The number of Oocysts Per Gram of excreta (OPG) in Experiment 2 was determined in excretal samples collected on days 5-7 PI. In Experiment 3 OPG was determined for days 5, 7, 9, 12, 14 and 19 PI. In Experiment 4 and 5, OPG was determined for days 6,13 and 21 PI. A modified McMaster counting chamber technique of Hodgson (1970) was used. A 10\% (w/v) excreta suspension in a salt solution (151 g NaCl mixed into $1 \mathrm{~L}$ of water) was prepared. After shaking thoroughly, $1 \mathrm{~mL}$ of the suspension was mixed with $9 \mathrm{~mL}$ of a salt solution (311 $\mathrm{g}$ of $\mathrm{NaCl}$ mixed into $1 \mathrm{~L}$ of water). Then, the suspension was put into the McMaster chamber using a micropipette and the number of oocysts was counted (Peek and Landman, 2003).

Lesion scoring: Lesion scoring in Experiment 1 and 2 was performed on day 6 PI, whereas in Experiment 3 and 4 it was done for days 14 and 19 PI and days 6 and 13 PI, respectively. In Experiment 5, lesion scoring was performed for days 6, 13 and $21 \mathrm{PI}$. The severity of coccidial lesions was scored while the investigator was blinded to treatment modality. In all experiments, the 0-4 scoring system described by Johnson and Reid (1970) was used. The lesions caused by the three species of Eimeria were monitored separately when applicable.

Statistical analysis: To identify statistically significant treatment effects, the technical data for each variable were subjected to the independent-samples $t$-Test procedure to compare the infected and uninfected means using SPSS (SPSS Inc, Chicago, USA). Prior to statistical testing, the oocyst values were logarithmically transformed $\left[\log _{10}(X+1)\right]$ to create a normal distribution and lesion scores were transformed using multinomial transformation. The level of statistical significance was pre-set at $\mathrm{p}<0.05$.

\section{RESULTS}

Growth performance: Body weight gain in all 5 experiments is illustrated in Table 2. In Experiment 1 and 2, there was no significant difference in weight gain between control and infected birds. Likewise, in Experiment 3 the birds infected by gavage on day 1 showed unaffected weight gain. The birds infected by gavage on day 15 with the three Eimeria species (Experiment 4) displayed a significant decrease in weight gain during the period of two weeks PI. In Experiment 5, the infection with the three Eimeria species had induced a significant decrease in weight gain, which was no longer apparent in the period of 1421 days PI.

In Experiment 1-3, feed intake and feed conversion ratios were similar for control and infected birds. In experiment 4, feed intake during days 1-6 PI was significantly lowered by the infection, but was unaffected thereafter. In Experiment 5, a significantly lower feed intake in the infected birds also was recorded for days 1-6 days PI. The feed conversion ratio for the infected birds was significantly higher during days 1-6 and 7-14 PI in experiment 4, but in Experiment 5 such an effect was not seen.

Lesion scores: In Experiment 1-3 the control birds were found to be free of lesions throughout the experiment. However, in Experiment 4 and 5 cross contamination had occurred (from day 13 PI and day 6 PI, respectively) which was indicated by lesion scores in the controls that were in general one unit lower than those seen in their infected counterparts. The lesion scores for the infected birds in all experiments are presented in Table 3. 
American J. Animal \& Vet. Sci., 5 (1): 45-51, 2010

Table 2: Post-Infection (PI) technical performance in the five experiments

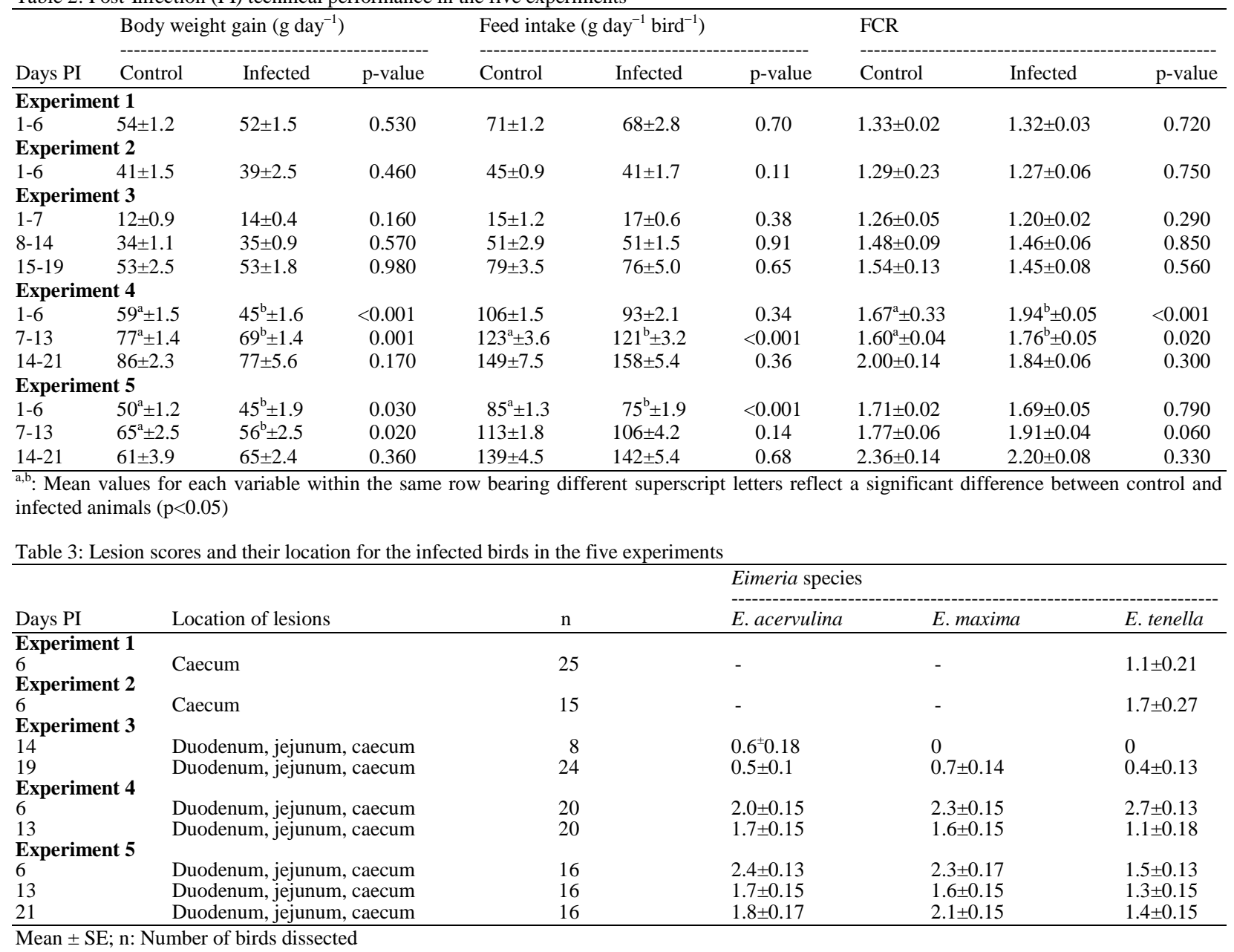

In Experiment 1 and 2 the caecal lesions scores were similar. For Experiment 3-5, the mean scores for the lesions in duodenum, jejunum and caecum are given. The small dose of oocysts used in Experiment 3 resulted on day 14 PI in an average duodenal lesion score of 0.6 for E. acervulina and absence of lesions due to E. maxima and E. tenella. On day 19 PI, lesions scores below 1 were seen for all three species of Eimeria. After infection with identical amounts of the three Eimeria species in Experiment 4 and 5, but administered through gavage or litter, respectively, similar lesion scores were observed in the infected birds. The scores all had values well above 1 .

Oocysts in excreta: OPG was not determined in Experiment 1. In Experiment 2, the OPG was measured in an excreta sample pooled for days 5-7 PI. In Experiment 3-5, oocyst shedding was followed during the time intervals indicated in Table 4.
Table 4: Number of Oocysts Per Gram excreta (OPG) expressed as $\log _{10}(\mathrm{X}+1)$ for the infected birds in the five experiments

\begin{tabular}{lcc}
\hline Days PI & $\mathrm{N}$ & $\begin{array}{l}\text { Oocysts Per Gram } \\
\text { excreta (OPG) }\end{array}$ \\
\hline Experiment 2 & & \\
5-7 & 5 & $4.80 \pm 0.28$ \\
Experiment 3 & 8 & $2.70 \pm 0.44$ \\
5 & 8 & $0.54 \pm 0.10$ \\
7 & 8 & $0.20 \pm 0.05$ \\
9 & 8 & $5.10 \pm 0.78$ \\
12 & 8 & $1.01 \pm 0.36$ \\
14 & 8 & $3.38 \pm 1.99$ \\
16 & 8 & $0.39 \pm 0.13$ \\
19 & 10 & \\
Experiment 4 & 10 & $5.70 \pm 0.35$ \\
6 & 10 & $4.02 \pm 0.34$ \\
13 & & $2.24 \pm 0.52$ \\
21 & 8 & $4.03 \pm 0.12$ \\
Experiment 5 & 8 & $4.58 \pm 0.20$ \\
6 & 8 & $3.82 \pm 0.22$ \\
13 & &
\end{tabular}


American J. Animal \& Vet. Sci., 5 (1): 45-51, 2010

The OPG pattern in Experiment 3 showed alternating peaks and dips. In Experiment 4 and 5 there were high OPG values on days 6 and 13 PI, followed by a fall on day 21 PI.

\section{DISCUSSION}

In all 5 experiments, there was a successful infection with Eimeria species as indicated by the intestinal lesions and the shedding of the oocysts. In Experiment 1-3 the infection had no influence on growth performance. The lack of effect of infection on growth performance may relate to the relatively low numbers of sporulated oocysts used. This observation corroborates earlier work (Conway et al., 1993). The higher numbers of oocysts used in Experiment 4 and 5 did indeed lower weight gain and feed intake PI. In Experiment 4, feed conversion was clearly increased by the infection. The impact of higher infection doses on weight gain and feed intake has also been reported by others (Barwick et al., 1970; Conway et al., 1993; Johnson and Reid, 1970). In Experiment 4 and 5 the reduction in the weight gain during days 1-6 PI was 24 and $13 \%$, respectively and the decrease in feed intake was $12 \%$ for both experiments. The similar effect of infection on feed intake in Experiment 4 and 5 may indicate that the route of the infection had no additional impact. Coccidiosis is an infectious disease that may reduce body-weight gain in broilers as a result of the combination of reduced feed intake and reduced digestibility and absorption of macronutrients (Adams et al., 1996).

The absence of the intestinal lesions due to E. maxima and E. tenella on day 14 PI in Experiment 3 may be explained by the low dose used and their low oocyst productive potential (Brackett and Bliznick, 1952). The lesion scores for E. acervulina and E. maxima in Experiment 4 and 5 were similar. This again indicates that the administration of equal doses of E. acervulina and E. maxima through either gavage or litter had no differential effect. However, in Experiment 5 the lesion scores due to E. tenella on day 6 PI were much lower than those seen in Experiment 4 on the same day PI. This difference may relate to the slow progress of the infection in Experiment 5 in which the oocysts were administered through the litter. The severity of the symptoms caused by $E$. tenella infection depends on the rate of the infection and the susceptibility of the birds. When the chickens gradually pick up the oocysts, they may become resistant before clinical effects appear as opposed to administration of a massive dose by gavage (Dalloul and Lillehoj, 2006).
In addition to growth performance and lesion scores, oocyst shedding also is a good index of the success of infection with the Eimeria. In Experiment 3 it was attempted to enhance immunity of the birds by the combination of an initial parasitic stage provided by an initial low coccidiosis infection dose and subsequent boosting the immunity by multiple re-infections with oocysts shed in the litter as does a non-attenuated vaccine (Chapman, 2000; Williams, 2002). Indeed, the pattern of oocyst excretion was as expected, mainly because the initial infection dose was low and more or less equal to the dose of a non-attenuated vaccine. Williams (2002) reported that in vaccinated birds, the oocyst stage of Eimeria that follow excystation initiates the vaccinal infection, hence stimulating immunity and that during subsequent recycling of infection this immunity is maintained. The phenomenon establishes 'trickle' infections that have been shown to be very effective in stimulating protective immunity (Joyner and Norton, 1973; 1976). Such infection model can be used to study the induction of the immunity by recycling of the infection in the presence of an agent with coccidiostatic activity. The prophylactic effects of such agents are obviously assisted by the chicken immune responses to coccidial challenges by oocyst accumulation in the litter (Chapman, 1999; Williams, 2002). Moreover, this model can be used to screen feed additives that have the potential to enhance the immunity of the host against Eimeria infection (Elmusharaf et al., 2007).

In Experiment 4 and 5, oocyst shedding on day 21 PI was lower than on days 6 and $13 \mathrm{PI}$, the difference being greater in Experiment 4. The high dose of the Eimeria mixture administered by gavage caused a high OPG value on day 6 PI followed by a steady reduction days 13 and $21 \mathrm{PI}$. This pattern may be explained by a crowding effect resulting from the boosting of the initial effect of the challenge dose by the secondary and the third infection produced by recycling of the oocysts shed in the litter (Brackett and Bliznick, 1952; Tyzzer et al., 1932; Williams, 2001; 2002). The pattern of oocyst shedding should be taken into account when interpreting the results of oocyst production in the testing of anticoccidial agents (Williams, 2001). The oocysts shedding pattern seen in Experiment 5 resembles that in field situations (Chapman, 2000). The infection through the litter on day 15 of the experiment resulted in a higher and lower oocyst shedding on days 13 and 21 PI respectively than that on day 6 PI. This observation is consistent with the outcome of an epidemiological survey showing that the oocysts in the litter or droppings of broiler chickens are usually most numerous at 4 weeks of age and generally 
decline as the birds become immune to further infection (Reyna et al., 1982; Williams et al., 1996). In the field, outbreaks of coccidiosis arise in one of three situations, that is in young, susceptible stock placed on litter in which a lethal concentration of oocysts is already present, in young stock placed on litter under conditions suitable for the sporulation and survival of oocysts so that the rate of infection is greater than the rate at which resistance develops and in adult stock which has been reared under conditions with minimal or no infection (Dalloul and Lillehoj, 2006). The infection model based on infected litter may be more reliable than a model based on one large single dose to individual birds. Williams (2002) reported that the use of a single massive level of inoculum in testing the efficacy of an anticoccidial agent may limit the ability to evaluate the expected challenge condition encountered in the field. A reduced sensitivity of Eimeria to some anticoccidials such as ionophores has been suspected for years (Chapman, 1982; Li et al., 2004; Stephan et al., 1997). The commercial success of ionophores indicates that the challenge conditions in the field are compatible with their different efficacy profiles (Bafundo and Jeffers, 1990; Chapman, 1984; Jeffers, 1978).

It is generally accepted that technical performance in particular weight gain, is the most sensitive and informative measure of anticoccidial efficacy (Barwick et al., 1970; Johnson and Reid, 1970; Long, 1970) and high levels of oocysts inocula are generally needed to achieve measurable suppression of weight gain in the infected unmedicated birds (Chapman, 1998; Conway et al., 1993). This would imply that the infection models used in Experiment 1-3 are unsuitable. However, the infection model used in Experiment 3 can be used to investigate the response of broiler chickens to immunostimulators when the criterion of efficacy is based on oocyst shedding and lesion scores. An additional, later challenge through the litter could be done to test for the immunity development and to evaluate the anticoccidial under investigation using lesion scores, oocyst shedding and technical performance as criterions.

\section{CONCLUSION}

Infection through gavage with a single high dose of inoculum has no similarity with the field situation and it might result in results with no practical relevance. Infection through the litter mimics the field situation in combination with controlled conditions and allowing experimental flexibility and a large number of experimental units within the research facility.

\section{REFERENCES}

Adams, C., A.A. Vahl and A. Veldman, 1996, Interaction between nutrition and $E$. acervulina infection in broilers chickens: diet compositions that improve fat digestion during $E$. acervulina infection. Br. J. Nutr., 75: 875-880. PMID: 8774232

Bafundo, K.W. and T.K. Jeffers, 1990. Selection for resistance to monensin, nicarbazin and the monensin plus nicarbazin combination. Poult. Sci., 69: 1485-1490. PMID: 2247409

Barwick, M.W., G.T. Stevenson, R.V. Johnston, D.R. Carsorso and T.A. Hymas, 1970. Coccidiosis: Evaluation of techniques for battery testing or field-collected Eimeria oocyst. Exp. Parasitol., 28: 37-41. PMID: 5459871

Brackett, S. and A. Bliznick, 1952. The reproductive potential of five species of coccidia of the chicken as demonstrated by oocyst production. J. Parasitol., 38: 133-139. PMID: 14946625

Chapman, H.D., 1982. The sensitivity of field isolates of Eimeria acervulina type to monensin. Vet. Parasitol., 9: 179-183. PMID: 7201192

Chapman, H.D., 1984: Eimeria tenella: Experimental development of resistance to monensin in the chicken. Parasitology, 89: 9-16. PMID: 6472888

Chapman, H.D., 1998. Evaluation of the efficacy of anticoccidial drugs against Eimeria species in the fowl. Int. J. Parasitol., 28: 1141-1144. DOI: 10.1016/S0020-7519(98)00024-1

Chapman, H.D., 1999. The development of immunity to Eimeria species in broilers given anticoccidial drugs. Avian Pathol., 28: 155-162. PMID: 16147557

Chapman, H.D., 2000. Practical use of vaccines for the control of coccidiosis in the chickens. World's Poult. Sci. J., 56: 7-20. ISSN: 0043-9339

Conway, D.P., K.S. Sasai, M. Gaafar and C.D. Smothers, 1993. Effects of different levels of oocyst inocula of Eimeria acervulina, E. tenella and E. maxima on plasma constituents, packed cell volume, lesion scores and performance in chickens. Avian Dis., 37: 118-123. PMID: 8452488

Dalloul, R.A. and H.S. Lillehoj, 2006. Poultry coccidiosis: Recent advancements in control measures and vaccine development. Expert Rev. Vaccines, 5: 143-63. PMID: 16451116

Elmusharaf, M.A., V. Bautista, L. Nollet and A.C. Beynen, 2006. Effect of a mannanoligosaccharide preparation on Eimeria tenella infection in broiler chickens. Int. J. Poult. Sci., 5: 583-588. 
Elmusharaf, M.A., J.J. Cuppen, H.N. Grooten and A.C. Beynen, 2007a. Antagonistic effect of electromagnetic field exposure on coccidiosis infection in broiler chickens. Poult. Sci., 86: 2139-2143. PMID: 17878443

Elmusharaf, M.A., H.W. Peek, L. Nollet and A.C. Beynen, 2007b. The effect of an in-feed Mannanoligosaccharide preparation (MOS) on a coccidiosis infection in broilers. Anim. Feed Sci. Technol., 134: 347-354. DOI: 10.1016/j.anifeedsci.2006.11.022

Hodgson, J.N., 1970. Coccidiosis: Oocyst counting technique for coccidiostat evaluation. Exp. Parasitol., 28: 99-102. PMID: 5459879

Jeffers, T.K., 1978: Eimeria tenella: sensitivity of recent field isolates to monensin. Avian Dis., 22: 157-161. PMID: 646756

Johnson, J. and W.M. Reid, 1970. Anticoccidial drugs: Lesion scoring techniques in battery and floor-pen experiments with chickens. Exp. Parasitol., 28: 30-36. PMID: 5459870

Joyner, L.P. and C.C. Norton, 1973. The immunity arising from continuous low-level infection with Eimeria tenella. Parasitology, 67: 333-340. PMID: 4761772

Joyner, L.P. and C.C. Norton, 1976. The immunity arising from continuous low-level infection with Eimeria maxima and Eimeria acervulina. Parasitology, 72: 115-125. PMID: 1256908

Li, G.Q., S.F. Kanu, Y. Xiang, S.M. Xiao and L. Zhang et al., 2004. Isolation and selection of ionophoretolerant Eimeria precocious lines: E. tenella, E. maxima and E. acervulina. Vet. Parasitol., 119: 261-276. PMID: 15154593

Long, P.L., 1970: Coccidiosis: Development of new techniques in coccidiostat evaluation. Exp. Parasitol., 28: 151-155. PMID: 5459864
Peek, H.W. and W.J.M. Landman, 2003. Resistance to anticoccidial drugs of Dutch avian Eimeria spp. field isolates originating from 1996, 1999 and 2001. Avian Pathol., 32: 391-401. PMID: 17585463

Reyna, P.S., G.F. Mathis and L.R. McDougald, 1982. Survival of coccidia in poultry litter and reservoirs of infection. Avian Dis., 27: 464-473.

Stephan, B., M. Rommel, A. Daugschies and A. Haberkorn, 1997. Studies of resistance to anticoccidials in Eimeria tenella field isolates and pure Eimeria strains. Vet. Parasitol., 69: 19-29. DOI: 10.1016/S0304-4017(96)01096-5

Tyzzer, E.E., H. Theiler and E.E. Jones, 1932. Coccidiosis in gallinaceous birds. II. A comparative study of species of Eimeria of the chickens. Am. J. Hygiene, 15: 319-393. PMID: 17797671

Williams, R.B., 2001. Quantification of the crowding effect during infections with the seven Eimeria species of the domestic fowl: Its importance for experimental designs and the production of oocyst stocks. Int. J. Parasitol., 31: 1056-1069. PMID: 11429169

Williams, R.B., 2002. Anticoccidial vaccines for broilers chickens: Pathway to success. Avian Pathol., 31: 317-353. PMID: 12396335

Williams, R.B., A.C. Bushell, J.M. Reperant, T.G. Doy and J.H. Morgan et al., 1996. A survey of Eimeria species in commercially reared chickens in France during 1994. Avian Pathol., 25: 113-130. PMID: 18645842 